\title{
Anatomic change over the course of treatment for non-small cell lung cancer patients and its impact on intensity- modulated radiation therapy and passive- scattering proton therapy deliveries
}

Mei Chen ${ }^{1,2}$, Jinzhong Yang ${ }^{2}$, Zhongxing Liao ${ }^{3}$, Jiayi Chen ${ }^{1}$, Cheng $\mathrm{Xu}^{1}$, Xiaodong $\mathrm{He}^{1}$, Xiaodong Zhang ${ }^{2}$, Ronald X. Zhu ${ }^{2}$ and Heng $\mathrm{Li}^{2,4^{*}}$ (D)

\begin{abstract}
Purpose: To quantify tumor anatomic change of non-small cell lung cancer (NSCLC) patients given passivescattering proton therapy (PSPT) and intensity-modulated radiation therapy (IMRT) through 6-7 weeks of treatment, and analyze the correlation between anatomic change and the need to adopt adaptive radiotherapy (ART).

Materials and methods: Weekly 4D CT sets of 32 patients (8/8 IMRT with/without ART, 8/8 PSPT with/without ART) acquired during treatment, were registered to the planning $\mathrm{CT}$ using an in-house developed deformable registration algorithm. The anatomic change was quantified as the mean variation of the region of interest (ROI) relative to the planning $C T$ by averaging the magnitude of deformation vectors of all voxels within the $\mathrm{ROI}$ contour. Mean variations of GTV and CTV were compared between subgroups classified by ART status and treatment modality using the independent $t$-test. Logistic regression analysis was performed to clarify the effect of anatomic change on the probability of ART adoption.

Results: There was no significant difference $(p=0.679)$ for the time-averaged mean CTV variations from the planning CT between IMRT $(7.61 \pm 2.80 \mathrm{~mm})$ and PSPT $(7.21 \pm 2.67 \mathrm{~mm})$ patients. However, a significant difference $(p=0.001)$ was observed between ART $(8.93 \pm 2.19 \mathrm{~mm})$ and non-ART $(5.90 \pm 2.33 \mathrm{~mm})$ patients, when treatment modality was not considered. Mean CTV variation from the planning CT in all patients increases significantly $(p<$ 0.001), with a changing rate of $1.77 \mathrm{~mm}$ per week. Findings for the GTV change was similar. The logistic regression model correctly predicted $71.9 \%$ of cases in ART adoption. The correlation is stronger in the PSPT group with a pseudo $R^{2}$ value of 0.782 , compared to that in the IMRT group (pseudo $R^{2}=0.182$ ).

(Continued on next page)
\end{abstract}

\footnotetext{
* Correspondence: Hengli@JHU.edu

${ }^{2}$ Department of Radiation Physics, The University of Texas MD Anderson Cancer Center, Houston, TX 77030, USA

${ }^{4}$ Department of Radiation Oncology and Molecular Radiation Sciences, Johns Hopkins University School of Medicine, Baltimore, MD 21205, USA

Full list of author information is available at the end of the article
}

(c) The Author(s). 2020 Open Access This article is licensed under a Creative Commons Attribution 4.0 International License, which permits use, sharing, adaptation, distribution and reproduction in any medium or format, as long as you give appropriate credit to the original author(s) and the source, provide a link to the Creative Commons licence, and indicate if changes were made. The images or other third party material in this article are included in the article's Creative Commons licence, unless indicated otherwise in a credit line to the material. If material is not included in the article's Creative Commons licence and your intended use is not permitted by statutory regulation or exceeds the permitted use, you will need to obtain permission directly from the copyright holder. To view a copy of this licence, visit http://creativecommons.org/licenses/by/4.0/ The Creative Commons Public Domain Dedication waiver (http://creativecommons.org/publicdomain/zero/1.0/) applies to the data made available in this article, unless otherwise stated in a credit line to the data. 
(Continued from previous page)

Conclusion: The magnitude of target volume variation over time could be greater than the usual treatment margin. Mean target volume variation from the planning position can be used to identify lung cancer patients that may need ART.

Keywords: Anatomic change, Proton therapy, Adaptive therapy

\section{Introduction}

External beam radiation therapy is an important technique in the management of lung cancer. Advanced beam delivery approaches such as intensity-modulated radiation therapy (IMRT) and proton therapy could improve dose conformality by creating a sharper dose gradient, which could, in turn, lead to reduced normaltissue dose and facilitating safe dose escalation within the tumor $[1,2]$. However, a sharper dose gradient demands a more precise dose delivery. Geometric uncertainties, especially intra-fractional tumor motion and inter-fractional anatomic changes, pose barriers to the accuracy of highly conformal treatment $[3,4]$. Reduction of geometric uncertainties is therefore imperative to improve the therapeutic ratio, and studies have suggested that the combination of advanced image guidance and motion management can improve local control and overall survival for patients with non-small cell lung cancer (NSCLC) $[5,6]$.

Major concerns remain for treating NSCLC patients using proton therapy due to the sensitivity of proton dose deposition toward any beam path-length change, which could be induced by intra- and inter-fractional motion for NSCLC patients [4]. The effect of intra-fractional tumor motion can be mitigated using pre-treatment fourdimensional (4D) computed tomography (CT) imaging and 4D CT-based treatment planning. Respirationcorrelated 4D CT shows the full range of possible positions of the tumor and critical organs, making it feasible to incorporate motion uncertainties into contouring, treatment planning, and treatment plan evaluation [7-9].

Adaptive radiotherapy (ART), which modifies the treatment plan to accommodate inter-fractional variation, is often used to mitigate uncertainties caused by anatomic changes. Dose benefits and better clinical outcomes were observed in lung cancer patients receiving ART than in those in a non-ART group $[10,11]$. ART for lung cancer was reported to reduce mean lung dose, volume of lung receiving 20 Gy (V20Gy), mean esophageal and heart dose, and maximum spinal cord dose [12, 13]. Compared with a non-ART group, the implementation of ART strategies including soft-tissue tumor matching for patients with locally advanced lung cancer increased the rate of local-regional control without increasing treatment-related toxicity [14]. The typical implementation of ART generally includes repeated CT simulation, re-delineation of structures, plan reoptimization, and successive plan review and approval, which increase the workload of therapists, dosimetrists, physicists, and physicians.

Schmidt et. al. compared the dosimetric impacts of respiratory motion, inter-fractional baseline shifts, and anatomic changes in NSCLC patients treated with IMRT using daily cone beam CT (CBCT) and mid-treatment 4D CT scans and found that anatomic changes had more impact on patient dose than did internal target motion. They also suggested that ART can be used to achieve better target coverage throughout the treatment course [15]. Therefore, it is important to identify patients who need ART and predict the optimal time in the course of treatment for implementing ART. While previous studies have focused on addressing the requirement for ART with regard to anatomic changes, predictors of the need for ART planning remain unclear, and ART adoption largely depends on physicians' discretion.

ART is used more often in proton therapy than in IMRT: 30\% of lung cancer patients who received proton therapy required ART, as opposed to $\sim 10 \%$ of those who received IMRT $[16,17]$. The greater need for ART in proton therapy could be due to the proton beams' depth dose characteristics and greater sensitivity to path length change compared with photon beams. However, the imaging data for proton radiotherapy patients is very limited compared to imaging data for photon radiotherapy patients, and there is a lack of quantitative studies comparing sensitivity towards anatomic change between proton and photon radiotherapy. The main purpose of the current study is to understand if there were any fundamental difference in terms of imaging response between proton and photon radiotherapy, and if patient imaging from photon radiation could be used for retrospective proton ART planning studies. To that end, we hypothesize that by delivering the same dose fractionation to the same target volume, there will be no observable imaging response difference using the two delivery techniques over a certain period of time during the treatment, with or without ART.

In addition, new planning techniques such as robust optimization and multiple CT optimization require a quantitative understanding of anatomic change so that these factors can be explicitly accounted for during planning [17-19]. If anatomic variations could be identified 
before treatment starts, or if there were a better understanding of the magnitude of anatomic change in the patient over time, then the initial treatment plan could be made more robust against subsequent anatomic change. However, accounting for the anticipated anatomic change in treatment planning is currently not feasible in clinical practice because information about the anatomic change embedded in the adaptive CT cannot be obtained until the patient is under treatment.

The current study aimed to bridge these knowledge gaps and identify predictors of the need for ART by quantifying the anatomic variations of patients treated with IMRT and passive-scattering proton therapy (PSPT) over the course of treatment and analyzing the correlation of these variations with ART adoption.

\section{Materials and methods Patient selection}

Repeated 4D CT sets were retrospectively reviewed for 32 lung cancer patients enrolled in a prospective randomized trial (NCT00915005), that compared the outcomes of PSPT and IMRT for inoperable NSCLC. All patients received radiotherapy at our institution during 2009 through 2014. Details of the trial have been recently published $[18,20]$. Of the 212 NSCLC patients enrolled in the trial, 136 received IMRT and 76 received PSPT. Each patient received weekly CT or CBCT (only available for IMRT patients) during their treatment. If the weekly scans showed a change in iGTV coverage (defined as $<95 \%$ target volume receiving $95 \%$ of the prescribed dose, or more than $2 \mathrm{~cm}^{3}$ receiving more than $120 \%$ of the prescribed dose), then ART was deemed necessary. Sixteen patients (12\%) who received IMRT and 22 patients (29\%) who received PSPT required at least one adaptive planning based on the weekly images. The rate of adaptive planning is consistent with other NSCLC patients treated at our center. Eight patients in the IMRT group and eight patients in the PSPT group required ART during their treatment were selected in this retrospective study. A same number of patients in the IMRT and PSPT arms, who did not need adaptive therapy, with GTV volume matched with the patients who needed adaptive therapy were also chosen for comparison. For each patient, the tumor was prescribed a total dose of 60 74 Gy (RBE) in 30-37 fractions over 6-7 weeks at 2 Gy (RBE) per fraction. Gy (RBE) is used in proton therapy prescription; it is the product of physical dose (Gy) and relative biologic effectiveness (RBE) for protons.

\section{Image acquisition}

Before treatment, all patients underwent 4D CT simulation for target delineation, motion assessment, and treatment planning. In addition to the pre-treatment planning 4D CT, each patient had 2-7 weekly 4D CTs.
The weekly CT was rigidly registered to the planning $\mathrm{CT}$ in a commercial treatment planning system (TPS, Pinnacle $^{3}$, Philips Healthcare, Andover, MA, for IMRT planning; Eclipse, Varian Medical Systems, for PSPT planning), and the original contours on the planning CT were then deformed to the weekly CT using an in house deformable image registration software [21]. A verification plan was generated by copying the treatment plan's beam data to the newly acquired CT, and the dose distribution was recalculated without optimization. If violation of dose constraints in target and/or normal tissues was observed, physicians requested ART on the weekly CT with new contours.

The 4D CT data sets were obtained in cine mode on a multislice CT scanner (Discovery ST or Lightspeed RT16, GE Healthcare, Chicago, IL). During each 4D CT scan, 10 three-dimensional (3D) CT sets reflecting 10 equally spaced phases during the breathing cycle were generated, along with maximum intensity projection (MIP) and average intensity projection (AIP) images. All images were uploaded to the PACS (picture archive and communication system) and then exported to the TPS.

\section{Target definition}

All the 3D CT sets reconstructed in one 4D CT scan were inherently registered to each other. The gross tumor volume (GTV) was initially delineated on MIP, T0 (end-inspiration, GTV_T0) and T50 (end-expiration, GTV_T50) phases; combined as internal gross tumor volume (IGTV); validated on all other phases; and then transferred to the AIP images for treatment planning. In the treatment plan of PSPT, the CT voxels inside the IGTV was overridden with a constant density to account for the intra-fractional motion effect [7]. The clinical target volume (CTV) was created by isotropically expanding the GTV by $8 \mathrm{~mm}$. Mean variations of the CTV on the T50 phase (CTV_T50, created by expanding GTV T50) were used for analyzing the deformation changes of the target during treatment.

\section{Image registration}

Images of the T50 phase of the weekly CT (moving CT) were registered to the planning CT (reference CT) using a rigid/deformable registration algorithm developed in house with MATLAB (Mathworks, Inc., Natick, MA). After the format consistency between the reference CT and moving $\mathrm{CT}$ was checked, rigid registration was performed to determine the isocenter shift between the reference CT and moving CT. The isocenter shift was then used to perform 3D Demons-based deformable registration [21], generating a deformation vector field between the reference $\mathrm{CT}$ and the moving $\mathrm{CT}$. 


\section{Quantification of anatomic change}

The variation between CTs for any given region of interest (ROI) was determined using all voxels of the deformation vector field within the ROI contour. For example, the weekly mean variations of the CTV relative to the initial CT were quantified as the mean value of the magnitude of the deformation vectors for all voxels within the CTV contours. Each vector magnitude is the square root of the sum of the squared $x$ (lateral), $y$ (anteriorposterior), $z$ (superior-inferior) displacement values. To evaluate the deformation trend between treatment modalities and ART status, weekly magnitudes averaged over the patients in each group were calculated. The rigid and deformable registration between $\mathrm{CT}$ data sets and data extraction for all patients were completed automatically via batch processing MATLAB code developed in house.

\section{Data analysis}

Differences in the mean variations of the CTV during the treatment were analyzed using a linear mixed-effects regression model that estimated patient variation as a random effect. Independent $t$-tests were performed to compare the mean CTV variations between

(1) patients with and without ART plans in the entire cohort,

(2) patients with and without ART plans in the IMRT group,
(3) patients with and without ART plans in the PSPT group,

(4) patients given IMRT and those given PSPT in the ART group, and

(5) patients given IMRT and those given PSPT in the non-ART group.

The statistical significance level was set at 0.05. All statistical analysis was done with SPSS version 24.0 (IBM, Chicago, IL). We performed comparisons 1, 2, and 3 to determine whether the mean CTV variation from planning CT was a good indicator of ART strategy. In addition, logistic regression was performed to ascertain the effect of tumor positional variations on the likelihood that patients use ART. By performing comparisons 4 and 5, we investigated the difference between two treatment technologies with regard to the tolerance of patient anatomical change as represented by mean CTV variations from the planning $\mathrm{CT}$.

\section{Results}

Patient data

The clinical characteristics of the patients are summarized in Table 1.

\section{Accuracy of deformable image registration}

The Demons-based deformable registration has been validated in multiple scenarios with reasonably good results [22-26]. Here, we illustrated the comparison of deformed contour and clinical approved contour for a

Table 1 Patients' clinical characteristics

\begin{tabular}{|c|c|c|c|}
\hline Characteristic & All $(n=32)$ & PSPT $(n=16)$ & $\operatorname{IMRT}(n=16)$ \\
\hline \multicolumn{4}{|l|}{ ART status, no. } \\
\hline With ART & 16 & 8 & 8 \\
\hline No ART & 16 & 8 & 8 \\
\hline 4DCT sets per patient, median (range) & $7(2-7)$ & $7(7-7)$ & $6.5(2-7)$ \\
\hline \multicolumn{4}{|l|}{ Sex, no. } \\
\hline Male & 19 & 11 & 8 \\
\hline Female & 13 & 5 & 8 \\
\hline Age, median (range), years & $64(43-78)$ & $64(43-76)$ & $62(47-78)$ \\
\hline \multicolumn{4}{|l|}{ Disease stage, no. } \\
\hline IV & 5 & 1 & 4 \\
\hline$\| \mathrm{IIB}$ & 21 & 12 & 9 \\
\hline$\| \mathrm{A}$ & 5 & 2 & 3 \\
\hline$\| \mathrm{A}$ & 1 & 1 & 0 \\
\hline \multicolumn{4}{|l|}{ Target volume, median (range), $\mathrm{cm}^{3}$} \\
\hline GTV & $221.9(12.2-686.6)$ & $127.1(22.7-673.7)$ & $280.9(12.2-686.6)$ \\
\hline CTV & $446.3(120.9-1329.3)$ & 398.7 (131.7-1329.3) & $558.2(120.9-1245.7)$ \\
\hline
\end{tabular}

IMRT intensity-modulated (photon) radiation therapy, PSPT passive-scattering proton therapy, GTV gross tumor volume, CTV clinical target volume, ART adaptive radiotherapy 
typical PSPT patient who underwent ART at week 4 in Fig. 1. The Dice similarity coefficient between the two contours shown in Fig. 1b is $93.68 \%$.

\section{Mean target variations from planning CT}

Figure 2 shows registration and analysis results for the same patient illustrated in Fig. 1. These results include anatomic changes over the course of 6 weeks of treatment (Fig. 2 a, as well as variations in the CTV, which changed drastically after week 4 , and in the spinal cord, which remained stable throughout the treatment (Fig. 2 b.

The mean GTV and CTV variations from the planning CT over time are shown in Table 2.

\section{Time trend of the mean target variations}

As treatment proceeded, both mean GTV and mean CTV variation from the planning CT increased significantly $(p<0.001)$, at a rate of 1.86 and $1.76 \mathrm{~mm}$ per week, respectively. The mean ( \pm standard deviation) initial variations (in week 1) for GTV and CTV in all patients were $4.85 \pm 2.03 \mathrm{~mm}$ (range $1.85-10.27 \mathrm{~mm}$ ) and $4.90 \pm 1.87 \mathrm{~mm}$ (range $2.01-9.44 \mathrm{~mm}$ ), respectively. The mean GTV and CTV variations in week 6 were $8.98 \pm$ $4.55 \mathrm{~mm}$ (range $3.23-21.35 \mathrm{~mm}$ ) and $8.50 \pm 4.18 \mathrm{~mm}$ (range $3.12-22.26 \mathrm{~mm}$ ), respectively. The time trend of the GTV and CTV variations did not differ significantly $(p=0.773)$, nor was there a significant difference between the time-averaged variations of the two ROIs $(p=$ 0.844). The weekly mean variation in CTV from the planning CT for all patient groups is shown in Fig. 3.

In both the ART and non-ART groups, the mean CTV variations significantly increased over weeks. The rate of increase in the ART group was $2.16 \mathrm{~mm}$ per week $(p<$ 0.001 ) and that in the non-ART group was $1.36 \mathrm{~mm}$ per week $(p<0.001)$. However, the rate of increase between the two groups is non-significantly different $(p=0.691)$.

\section{Time-averaged mean target variations}

Table 3 shows the comparison of time-averaged mean GTV and CTV variations from the planning CT between the ART and non-ART groups in the whole cohort and in modality-stratified subgroups.

Again, the results for GTV and CTV are similar. Overall, patients with ART plans showed a significantly larger displacement, at $9.41 \pm 2.17 \mathrm{~mm}$ for GTV and $8.93 \pm 2.19 \mathrm{~mm}$ for CTV, respectively, than did patients without ART plans, whose displacement was $6.07 \pm 2.62 \mathrm{~mm}$ for GTV and $5.90 \pm 2.33 \mathrm{~mm}$ for CTV $(p=0.001)$, respectively, without taking treatment modality into consideration. The minimum CTV shifts were $3.43 \mathrm{~mm}$ in the ART group and $6.47 \mathrm{~mm}$ in the non-ART group. Similarly, in the PSPT group, CTV variation from planning CT significantly differed between patients with and without ART plans $(p<$ 0.001). However, in the IMRT group, in which mean CTV variations from the planning CT were $6.61 \pm 2.82 \mathrm{~mm}$ in the ART group and $8.63 \pm 2.57 \mathrm{~mm}$ in the non-ART group, the difference was non-significant $(p=0.156)$. Nor did the IMRT and PSPT subgroups significantly differ with regard to CTV variation from the planning CT in either the ART $(p=0.596)$ or non-ART $(p=0.241)$ cohort.

The time-averaged mean CTV variation from the planning CT was $7.41 \pm 2.71 \mathrm{~mm}$ among all patients. These variations did not significantly differ between patients given IMRT $(7.61 \pm 2.80 \mathrm{~mm})$ and those given PSPT $(7.21 \pm 2.67$ $\mathrm{mm} ; p=0.679$ ), regardless of ART status, indicating that the magnitude of patient anatomic change over the course of treatment was similar across treatment modalities.

\section{Correlation of mean CTV variation with ART adoption}

Figure 4 shows the logistic regression curves for the entire cohort, the PSPT group, and the IMRT group.

The figure shows a statistically significant relationship between an increasing mean CTV variance from planning (a) Week 0

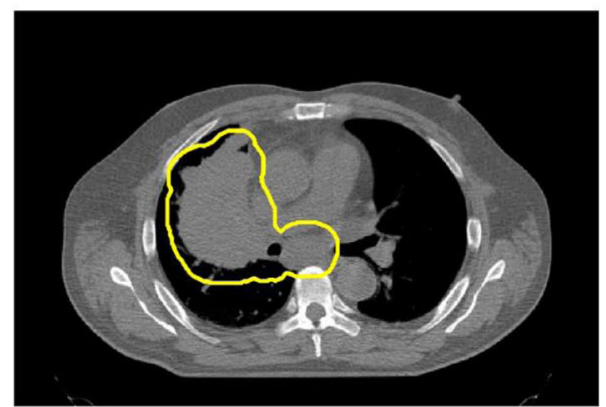

(b) Week 4

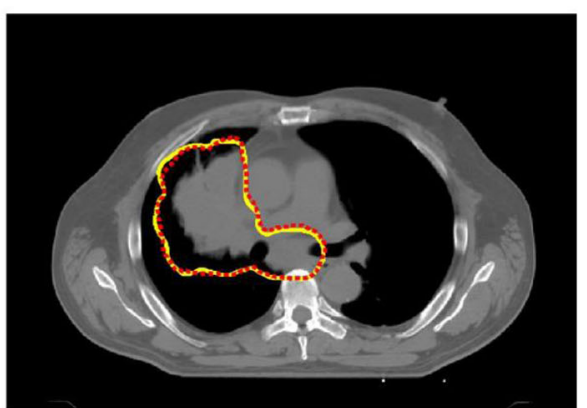

Fig. 1 Clinical target volume (CTV) contour on the CT acquired at weeks 0 (planning CT) a for a typical patient enrolled in the passive scattering proton therapy (PSPT) group, who received adaptive radiotherapy (ART) at week 4 . The deformed contour (red dash line) was compared with the clinical approved contour on the CT acquired at week $4 \mathbf{b}$ 


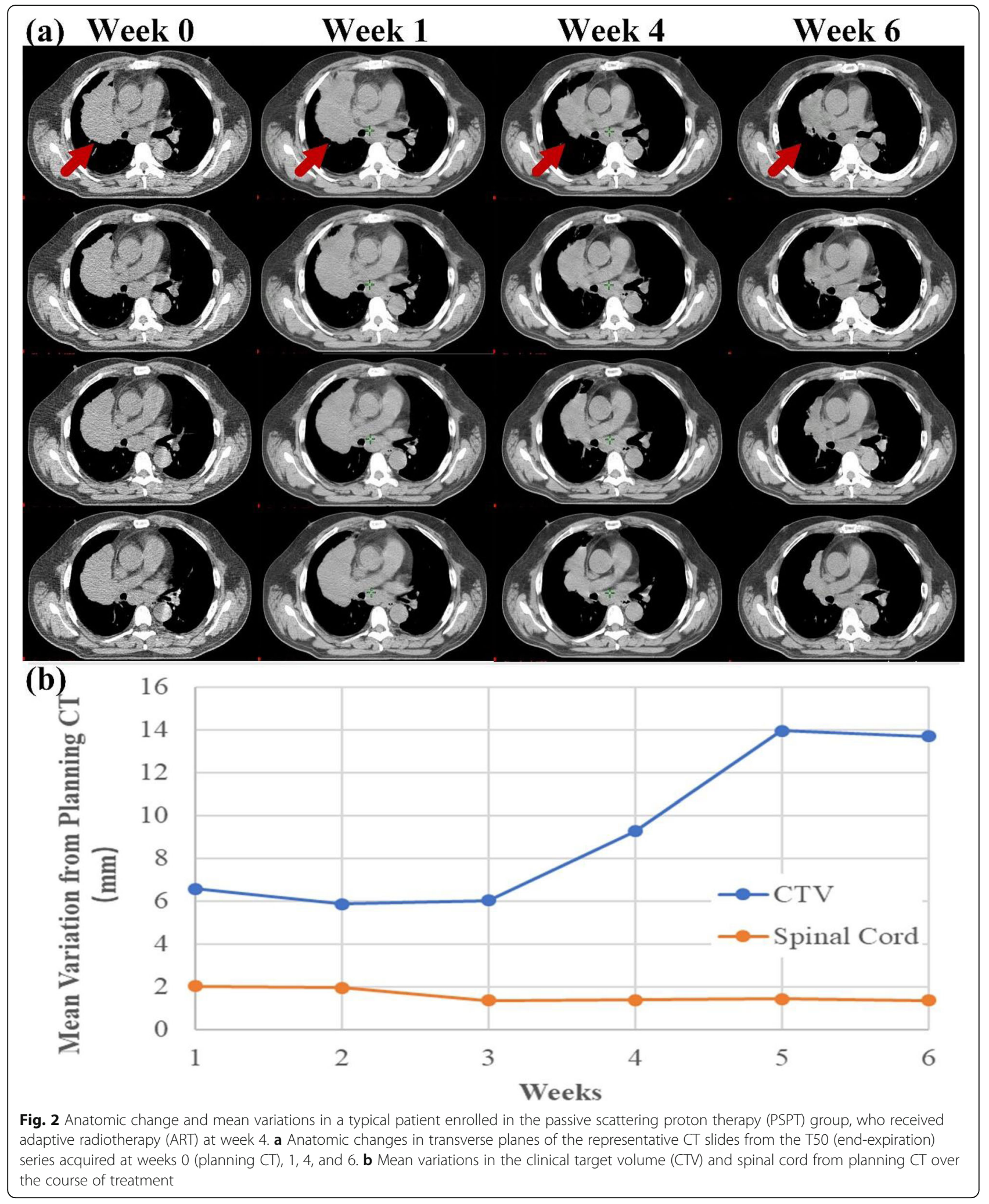


Table 2 Mean CTV variations from the planning CT over time for the entire cohort

\begin{tabular}{|c|c|c|c|c|c|c|c|}
\hline & Weeks (deviations in mm) & $1(\mathrm{~mm})$ & $2(\mathrm{~mm})$ & $3(\mathrm{~mm})$ & $4(\mathrm{~mm})$ & $5(\mathrm{~mm})$ & $6(\mathrm{~mm})$ \\
\hline \multirow[t]{3}{*}{ GTV } & Entire cohort & $4.85 \pm 2.03$ & $6.61 \pm 3.01$ & $7.06 \pm 3.23$ & $7.92 \pm 3.78$ & $8.19 \pm 4.18$ & $8.98 \pm 4.55$ \\
\hline & With ART & $5.98 \pm 2.17$ & $7.58 \pm 2.82$ & $9.04 \pm 3.23$ & $9.91 \pm 3.04$ & $10.31 \pm 3.47$ & $11.46 \pm 4.54$ \\
\hline & No ART & $3.86 \pm 1.28$ & $5.65 \pm 3.01$ & $5.20 \pm 1.88$ & $6.06 \pm 3.50$ & $6.34 \pm 3.92$ & $6.81 \pm 3.38$ \\
\hline \multirow[t]{3}{*}{ CTV } & Entire cohort & $4.90 \pm 1.90$ & $6.39 \pm 2.82$ & $6.87 \pm 3.06$ & $7.51 \pm 3.48$ & $7.79 \pm 3.88$ & $8.50 \pm 4.18$ \\
\hline & With ART & $5.99 \pm 1.97$ & $7.22 \pm 2.62$ & $8.56 \pm 3.27$ & $9.32 \pm 2.81$ & $9.75 \pm 3.37$ & $10.71 \pm 4.45$ \\
\hline & No ART & $3.94 \pm 1.24$ & $5.66 \pm 2.87$ & $5.28 \pm 1.79$ & $5.81 \pm 3.24$ & $6.08 \pm 3.54$ & $6.56 \pm 2.84$ \\
\hline
\end{tabular}

GTV internal gross tumor volume, CTV clinical target volume, $A R T$ adaptive radiotherapy

$\mathrm{CT}$ and an increasing likelihood of ART treatment for the entire cohort $(p=0.006)$. The model correctly predicted $71.9 \%$ of cases and explained $42.8 \%$ of the variance in ART strategy adoption. The correlation was stronger in the PSPT group (pseudo $\mathrm{R}^{2}=0.782$ ) than in the IMRT group (pseudo $\mathrm{R}^{2}=0.182$ ). A higher incidence of ART planning was observed in week 4 , accounting for more than half of the cases.

\section{Discussion}

In this quantitative study of relationship between ART and magnitude of inter-fractional anatomic variation over the course of treatment, the time-averaged mean CTV variations from the planning CT did not significantly differ between the IMRT $(7.61 \pm 2.80 \mathrm{~mm})$ and PSPT $(7.21 \pm 2.67 \mathrm{~mm})$ groups, but did significantly differ between the ART $(8.93 \pm 2.19 \mathrm{~mm})$ and non-ART $(5.90 \pm$
$2.33 \mathrm{~mm}$ ) groups. These results indicate that patient anatomy changes substantially over the course of treatment regardless of treatment modality and provide a quantitative foundation for improving treatment planning techniques. Our results also suggests that it is indeed feasible to use imaging data from photon radiotherapy patients, which is more readily available, for retrospective proton ART planning studies, as there is no significant difference was identified between the imaging responses of the target in patients treated the two different modalities.

We also investigated the influence of anatomic change on the need for ART in the context of treatment modality. Among the patients given IMRT, mean CTV variations from the planning CT did not significantly differ between the ART and non-ART groups, suggesting that IMRT is more robust to anatomic changes than is PSPT.

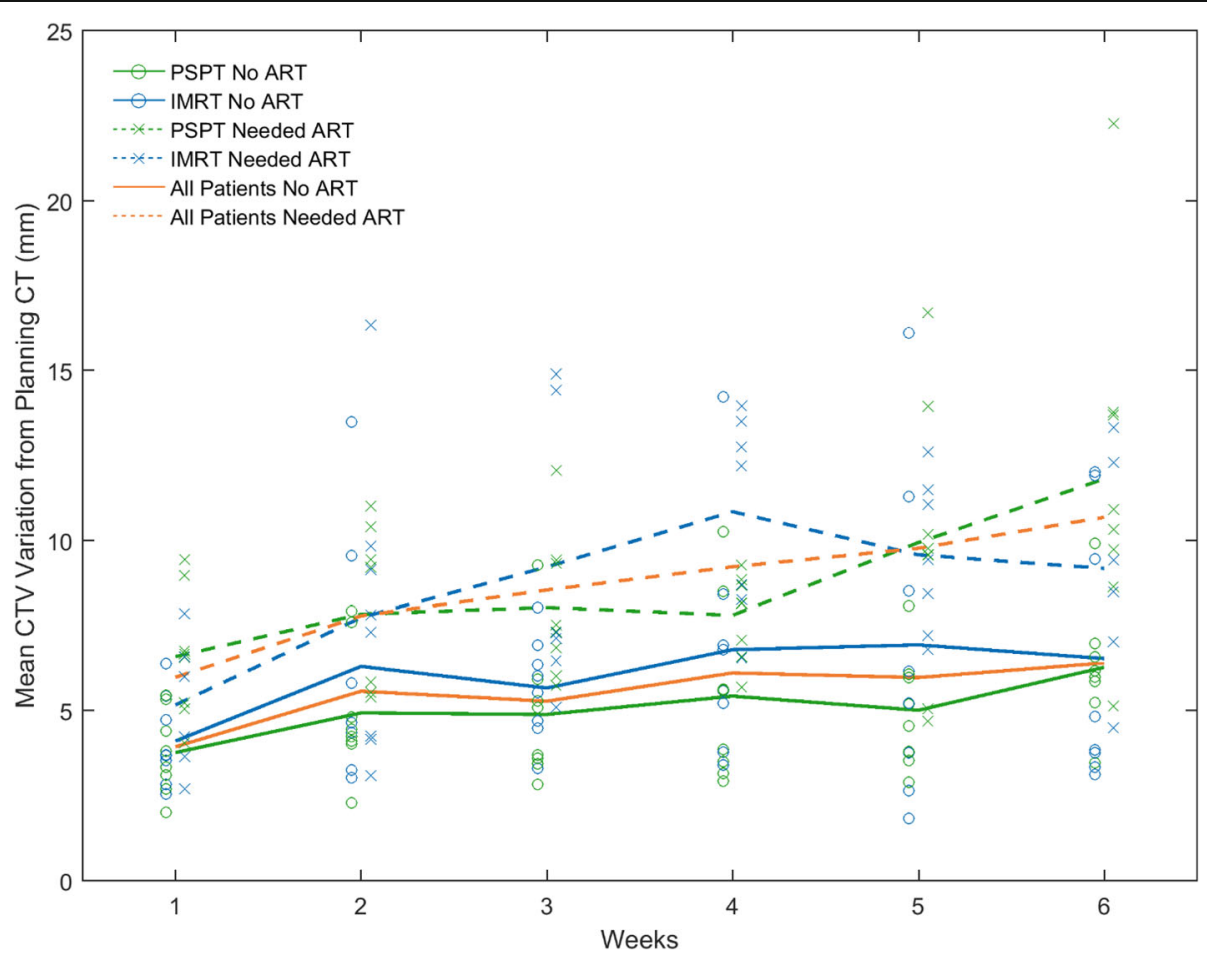

Fig. 3 Mean CTV variations each week for the entire cohort, the PSPT group, and the intensity-modulated radiation therapy (IMRT) group 
Table 3 Time-averaged mean GTV and CTV variations from the planning $C T$ within different groups

\begin{tabular}{lllll}
\hline \multicolumn{1}{c}{ Group } & Non-adaptive $(\mathrm{mm})$ & Adaptive $(\mathrm{mm})$ & $p$ value \\
\hline GTV & Entire cohort & $6.07 \pm 2.63$ & $9.41 \pm 2.17$ & 0.001 \\
& PSPT & $5.20 \pm 1.66$ & $9.76 \pm 2.01$ & $<0.001$ \\
& IMRT & $6.94 \pm 3.21$ & $9.06 \pm 2.41$ & 0.159 \\
CTV & Entire cohort & $5.90 \pm 2.33$ & $8.93 \pm 2.19$ & 0.001 \\
& PSPT & $5.19 \pm 1.59$ & $9.24 \pm 1.85$ & $<0.001$ \\
& IMRT & $6.61 \pm 2.82$ & $8.63 \pm 2.57$ & 0.156
\end{tabular}

GTV internal gross tumor volume, CTV clinical target volume, IMRT intensitymodulated (photon) radiation therapy, PSPT passive-scattering proton therapy

It is essential to adapt treatment planning to tumor motion and anatomic change in radiation treatment for lung cancer. Unlike respiratory motion, which can be incorporated in the initial treatment plan via 4D CT, anatomic change can currently be compensated for only by adaptive re-planning on a new CT. Moreover, compared with photon therapy, proton radiotherapy is more sensitive to density variation in the beam path. In the presence of setup uncertainties and anatomic changes, a higher frequency than photon therapy of re-imaging and re-planning has been recommended in proton therapy [16]. There are $\sim 30 \%$ of lung cancer patients who received proton therapy required ART, as opposed to $10 \%$ of those who received photon therapy [16, 17, 27].
However, the optimal timing of ART for each treatment technique with regard to anatomic change is unknown. In addition, the initial treatment plan with robust optimization may not be able to account for anatomic variations induced by treatment [17]. To ensure tumor coverage and normal tissue sparing, patients must undergo periodic CT imaging, and clinical staff, including a dosimetrist, a physicist, and a physician, must work on several verification plans on the repeated CT set regardless of treatment technique.

In previous studies, anatomic change was commonly quantified by tumor volume on repeated CT images, which could be performed using fluorodeoxyglucose positron emission tomography (FDG PET)/CT, CBCT, megavoltage $\mathrm{CT}$, or $4 \mathrm{D} \mathrm{CT}$. The target was recontoured on every periodic image set by physicians. The additional delineation was not only time-consuming but also found to be associated with additional uncertainty [28]. We demonstrated the correlation of ART strategy use with anatomic change in the tumor, characterized as the mean CTV deviation on weekly 4D CT imaging. CTV displacement was calculated by averaging the magnitude of the deformation vector rather than relying on target contours. Both the magnitude of anatomic change and the rate of change over time for CTV volume were much greater than those of a more stable volume, e.g., spinal cord (Fig. 2b). While the CTV

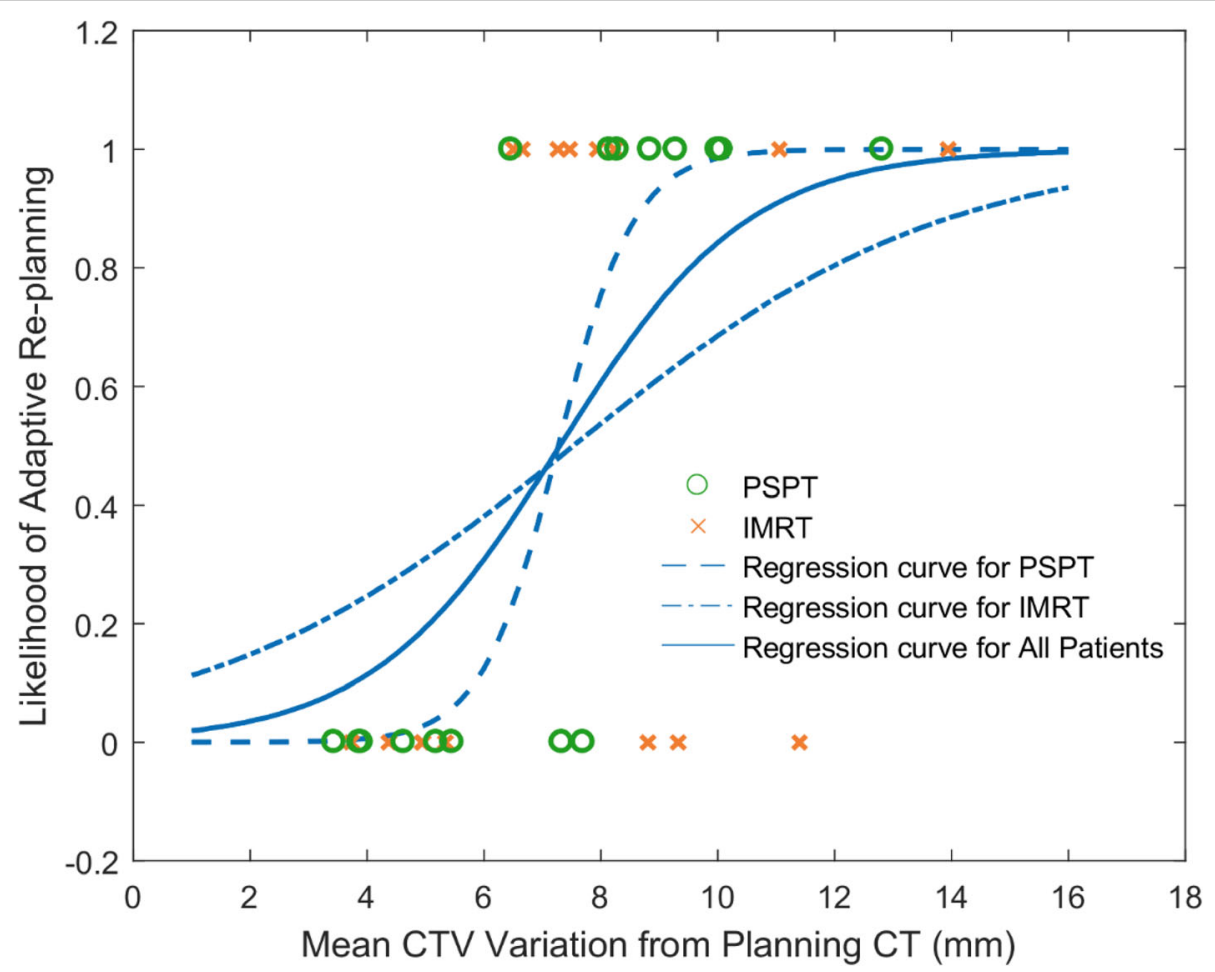

Fig. 4 Likelihood of adaptive re-planning versus time-averaged mean CTV variance from planning $C T$. The $S$ curves are the logical regression functions fitted to the binary data for all patients, the PSPT group, and the IMRT group 
change for patients in the non-ART group $(5.90 \pm 2.33$ $\mathrm{mm}$ ) was close to our usual planning margin with image guidance (planning target volume or CTV to PTV expansion, and setup uncertainty of $5 \mathrm{~mm}$ for IMRT and PSPT, respectively), the CTV change in the adaptive group was clearly more than the established planning margins; therefore, new planning techniques are needed to account for these changes.

Mixed-effects linear regression showed that mean CTV variation from the planning CT increased significantly over the course of treatment, at $1.77 \mathrm{~mm}$ per week. The rate of increase in the ART group was higher than that in the non-ART group. Similar time trends were seen in patients given IMRT and those given PSPT. These results are similar to the findings of Britton et al. [29], who observed a significant $(p=0.049)$ increase over the course of treatment in GTV and internal target volume centroid variation in a retrospective study of eight patients with non-small cell lung cancer, in which weekly $\mathrm{CT}$ was rigidly registered to planning $\mathrm{CT}$ and the position of the target centroid was measured on every image set. Hassbeek et al. [30] and Sun et al. [31], using a similar method but confined to a short treatment course of hyperfractional radiotherapy, reported a limited time trend in the mean 3D displacement of the PTV and GTV centroid. However, these previous studies captured only the tumor contour changes over time and disregarded tumor density variation and changes outside of the target, which could both be relevant to radiotherapy, especially proton therapy. In the current study, we used deformable registration to acquire patient anatomic change over time for each voxel and thus were able to capture tumor deformation information, such as tumor shrinkage, growth, and displacement, that may not be feasible to measure using centroid position changes only.

The action level of ART adoption varies with institutional practice and treatment delivery technique. Van den Bosch et al. [32] developed an automatic method to select patients eligible for ART with an accuracy of $79 \%$. The criterion set was the change in the water-equivalent path length to the edge of the target on daily CBCT. In the present, single-center study, mean CTV variation from the planning $\mathrm{CT}$ was found to significantly differ between ART and non-ART groups on an independent $t$-test, which indicates that mean CTV variation from the planning CT could be a good predictor of the need for ART. The correlation coefficient between CTV variation from the planning CT and ART treatment was 0.43 , and the predictive accuracy of the logistic regression model was $71.9 \%$. Plan updates may be warranted when the 3D shift of mean CTV variation from the planning CT exceeds $8.26 \mathrm{~mm}$, the median such variance for the ART group. Studies by Berkovic et al. [33] and Britton et al. [29] suggested that the optimal time point for
ART was around fraction 15, after week 3 . However, in our study, up to $60 \%$ of the ART re-planning occurred in week 4. ART decision-making is affected by multiple parameters, including patient weight change, pleural effusion, atelectasis, dose-volume histogram, dose distribution, potential clinical gain, and availability of clinical resources. Additional evidence is required to back any single indicator for formulating a patient-specific ART strategy. Correlations between dose change, treatment outcome, and anatomic variation need to be investigated in further studies.

Due to the purpose of the study, patient selection was difficult due to the limited patient size in the ART arm, and the different percentage of patients required ART between IMRT and PSPT modalities. Since the ART status was unknown when the patient was randomized to receive IMRT or PSPT, the anatomy change should not be dependent on treatment modality, which was confirmed by our results. We acknowledged that the effectiveness of current approach is limited by the registration error of the deformable image registration. The Demons registration algorithm used in this study has been fully validated for $4 \mathrm{D}$ CT registration. In our previous study, the mean registration accuracy of 300 landmark pairs was within $1.3 \mathrm{~mm}$ on ten $4 \mathrm{D}$ CT datasets from deformable image registration lab (http://dir-lab.com) [34]. This algorithm has also achieved registration accuracy of within $1.1 \mathrm{~mm}$ when comparing the calculated deformation and the known deformation in the voxel-level validation [24]. The performance of deformable image registration varied with registration scenarios and may not work properly in the presence of unexpected anatomy changes. For all registrations in the work, we randomly spot checked one registration for each patient using the overlapped contours to ensure no gross registration errors. Also, it worth mentioning that, deformable registration is an ill-posed problem with multiple solutions. The registration results rely on the underlying assumption of the regularization on the registration. The voxel-level deformation vectors might not reflect the actual anatomy change at the voxel location due to uncertainties; however, using the mean of deformation vectors inside an ROI could minimize the impact from uncertainties, thus approximating the actual anatomy changes of the ROI, as what we did in this project. Another limitation of the current study is that only inter-fractional changes in the patient were investigated. Although previous studies showed intra-fractional respiratory motion produces only relatively minor variations, it could still bring about dosimetric changes, which is the main clinical criterion for ART decision-making. Refraining from considering intra-fractional variations would slightly weaken the ability of inter-fractional changes to predict ART need. A more straightforward and effective method 
for identifying patients needing ART would be to explore the quantitative dose variation of the target and normal tissues in a large cohort on the basis of the deformable registration methods presented in this study.

\section{Conclusion}

The magnitude of CTV variation over time could be greater than the usual treatment margin. Mean CTV deviation from the planning position can be used to identify lung cancer patients who need ART. This indicator has stronger predictive power for ART decision-making in patients receiving PSPT than in those receiving IMRT. Further research into image and dose prediction is needed for the efficient implementation of ART.

\begin{abstract}
Abbreviations
4D CT: Four-dimensional computed tomography; AIP: Average intensity projection; ART: Adaptive radiotherapy; CBCT: Cone beam computed tomography; CTV: Clinical target volume; GTV: Gross tumor volume; IGTV: Internal gross tumor volume; IMRT: Intensity-modulated radiation therapy; MIP: Maximum intensity projection; NSCLC: Non-small cell lung cancer; PSPT: Passive-scattering proton therapy; RBE: Relative biologic effectiveness; ROI: Region of interest
\end{abstract}

\section{Acknowledgments}

We thank Bronson, Sarah J in M.D. Anderson's Department of Scientific Publications for the editorial review of this manuscript.

\section{Authors' contributions}

Study design: HL, JY, RZ, ZL. Data collection and analysis: MC, HL. Manuscript preparation: MC. Manuscript review and editing: HL, ZL, JC, CX, XH, XZ. All authors read and approved the final manuscript.

\section{Funding}

None.

\section{Availability of data and materials}

The datasets related to patient information are not available. Other data used and/or analyzed during the current study are available from the corresponding author on reasonable request.

\section{Ethics approval and consent to participate}

The protocol of this retrospective study was reviewed and approved by the IRB of the University of Texas MD Anderson Cancer Center.

\section{Consent for publication}

Non-applicable.

\section{Competing interests}

The authors declare that they have no competing interests.

\section{Author details}

'Department of Radiation Oncology, Ruijin Hospital, Shanghai Jiao Tong University School of Medicine, Shanghai 200025, China. ${ }^{2}$ Department of Radiation Physics, The University of Texas MD Anderson Cancer Center, Houston, TX 77030, USA. ${ }^{3}$ Department of Radiation Oncology, The University of Texas MD Anderson Cancer Center, Houston, TX 77030, USA. ${ }^{4}$ Department of Radiation Oncology and Molecular Radiation Sciences, Johns Hopkins University School of Medicine, Baltimore, MD 21205, USA.

Received: 16 April 2019 Accepted: 19 February 2020

Published online: 05 March 2020

\section{References}

1. Chang JY, Zhang $X$, Wang $X$, et al. Significant reduction of normal tissue dose by proton radiotherapy compared with three-dimensional conformal or intensity-modulated radiation therapy in stage I or stage III non-small-cell lung cancer. Int J Radiat Oncol Biol Phys. 2006;65(4):1087-96.

2. Chun SG, Hu C, Choy H, et al. Impact of intensity-modulated radiation therapy technique for locally advanced non-small-cell lung Cancer: a secondary analysis of the NRG oncology RTOG 0617 randomized clinical trial. J Clin Oncol. 2017:35(1):56-62.

3. Korreman SS. Motion in radiotherapy: photon therapy. Phys Med Biol. 2012; 57(23):R161-91.

4. Bert C, Durante M. Motion in radiotherapy: particle therapy. Phys Med Biol. 2011;56(16):R113-44.

5. Korreman SS. Image-guided radiotherapy and motion management in lung cancer. Br J Radiol. 2015;88(1051):20150100.

6. Kilburn JM, Soike MH, Lucas JT, et al. Image guided radiation therapy may result in improved local control in locally advanced lung cancer patients. Pract Radiat Oncol. 2016;6(3):e73-80.

7. Kang $Y$, Zhang $X$, Chang JY, et al. $4 D$ proton treatment planning strategy for mobile lung tumors. Int J Radiat Oncol Biol Phys. 2007:67(3):906-14.

8. Ezhil M, Vedam S, Balter P, et al. Determination of patient-specific internal gross tumor volumes for lung cancer using four-dimensional computed tomography. Radiat Oncol. 2009;4:4.

9. Yu J, Zhang X, Liao L, et al. Motion-robust intensity-modulated proton therapy for distal esophageal cancer. Med Phys. 2016;43(3):1111-8.

10. Gomez DR, Chang JY. Adaptive radiation for lung cancer. J Oncol. 2011; 2011:898391.

11. Sonke JJ, Belderbos J. Adaptive radiotherapy for lung cancer. Semin Radiat Oncol. 2010;20(2):94-106.

12. Ramsey CR, Langen KM, Kupelian PA, et al. A technique for adaptive imageguided helical tomotherapy for lung cancer. Int J Radiat Oncol Biol Phys. 2006;64(4):1237-44.

13. Kataria T, Gupta D, Bisht SS, et al. Adaptive radiotherapy in lung cancer: dosimetric benefits and clinical outcome. Br J Radiol. 2014;87(1038): 20130643.

14. Tvilum M, Khalil AA, Moller DS, Hoffmann L, Knap MM. Clinical outcome of image-guided adaptive radiotherapy in the treatment of lung cancer patients. Acta Oncol. 2015;54(9):1430-7.

15. Schmidt ML, Hoffmann L, Kandi M, Moller DS, Poulsen PR. Dosimetric impact of respiratory motion, interfraction baseline shifts, and anatomical changes in radiotherapy of non-small cell lung cancer. Acta Oncol. 2013; 52(7):1490-6.

16. Chang JY, Li H, Zhu XR, et al. Clinical implementation of intensity modulated proton therapy for thoracic malignancies. Int J Radiat Oncol Biol Phys. 2014;90(4):809-18.

17. Li H, Zhang $X$, Park $P$, et al. Robust optimization in intensity-modulated proton therapy to account for anatomy changes in lung cancer patients. Radiother Oncol. 2015;114(3):367-72.

18. Yang $\mathrm{P}, \mathrm{X} \cup \mathrm{T}, \mathrm{Gomez} \mathrm{DR}$, et al. Patterns of local-regional failure after intensity modulated radiation therapy or passive scattering proton therapy with concurrent chemotherapy for non-small cell lung Cancer. Int J Radiat Oncol Biol Phys. 2019;103(1):123-31.

19. Wang X, Li H, Zhu XR, et al. Multiple-CT optimization of intensity-modulated proton therapy - is it possible to eliminate adaptive planning? Radiother Oncol. 2018;128(1):167-73.

20. Liao Z, Lee JJ, Komaki R, et al. Bayesian adaptive randomization trial of passive scattering proton therapy and intensity-modulated photon radiotherapy for locally advanced non-small-cell lung Cancer. J Clin Oncol. 2018;36(18):1813-22

21. Wang $H$, Garden $A S$, Zhang $L$, et al. Performance evaluation of automatic anatomy segmentation algorithm on repeat or four-dimensional computed tomography images using deformable image registration method. Int J Radiat Oncol Biol Phys. 2008;72(1):210-9.

22. Mohamed ASR, Ruangskul M-N, Awan MJ, et al. Quality assurance assessment of diagnostic and radiation therapy-simulation CT image registration for head and neck radiation therapy: anatomic region of interest-based comparison of rigid and deformable algorithms. Radiology. 2015;274(3):752-63.

23. Zhang YB, Yang JZ, Zhang LF, et al. Digital reconstruction of high-quality daily $4 D$ cone-beam $C T$ images using prior knowledge of anatomy and respiratory motion. Comput Med Imaging Graph. 2015;40:30-8.

24. Yu ZH, Kudchadker R, Dong $L$, et al. Learning anatomy changes from patient populations to create artificial $\mathrm{CT}$ images for voxel-level validation of deformable image registration. J Appl Clin Med Phys. 2016;17(1):246-58. 
25. Yang JZ, Zhang YB, Zhang ZJ, Zhang LF, Batter P, Court L. Technical note: density correction to improve $C T$ number mapping in thoracic deformable image registration. Med Phys. 2019;46(5):2330-6.

26. Wang $\mathrm{H}$, Dong L, O'Daniel J, et al. Validation of an accelerated 'demons' algorithm for deformable image registration in radiation therapy. Phys Med Biol. 2005;50(12):2887-905.

27. Yang P, Xu T, Gomez DR, et al. Patterns of local-regional failure after intensity-modulated radiation therapy or passive scattering proton therapy with concurrent chemotherapy for non-small cell lung Cancer. Int J Radiat Oncol Biol Phys. 2018. https://doi.org/10.1016/j.jirobp.2018.08.031.

28. Van de Steene J, Linthout N, de Mey J, et al. Definition of gross tumor volume in lung cancer: inter-observer variability. Radiother Oncol. 2002; 62(1):37-49.

29. Britton KR, Starkschall G, Tucker SL, et al. Assessment of gross tumor volume regression and motion changes during radiotherapy for non-small-cell lung cancer as measured by four-dimensional computed tomography. Int J Radiat Oncol Biol Phys. 2007;68(4):1036-46.

30. Haasbeek CJ, Lagerwaard FJ, Cuijpers JP, Slotman BJ, Senan S. Is adaptive treatment planning required for stereotactic radiotherapy of stage I nonsmall-cell lung cancer? Int J Radiat Oncol Biol Phys. 2007;67(5):1370-4.

31. Sun $Y, G e H$, Cheng $S$, et al. Evaluation of interfractional variation of the centroid position and volume of internal target volume during stereotactic body radiotherapy of lung cancer using cone-beam computed tomography. J Appl Clin Med Phys. 2016;17(2):461-72.

32. van den Bosch M, Öllers M, Reymen B, van Elmpt W. Automatic selection of lung cancer patients for adaptive radiotherapy using cone-beam CT imaging. Phys Imaging Radiat Oncol. 2017;1:21-7.

33. Berkovic $P$, Paelinck $L$, Lievens $Y$, et al. Adaptive radiotherapy for locally advanced non-small cell lung cancer, can we predict when and for whom? Acta Oncol. 2015;54(9):1438-44.

34. Z Zhang YB, Yang JZ, Zhang LF, Court LE, Balter PA, Dong L. Modeling respiratory motion for reducing motion artifacts in $4 \mathrm{D} C \mathrm{CT}$ images. Med Phys. 2013:40(4):041716.

\section{Publisher's Note}

Springer Nature remains neutral with regard to jurisdictional claims in published maps and institutional affiliations.

Ready to submit your research? Choose BMC and benefit from:

- fast, convenient online submission

- thorough peer review by experienced researchers in your field

- rapid publication on acceptance

- support for research data, including large and complex data types

- gold Open Access which fosters wider collaboration and increased citations

- maximum visibility for your research: over $100 \mathrm{M}$ website views per year

At $\mathrm{BMC}$, research is always in progress.

Learn more biomedcentral.com/submissions 